\title{
Hochdeutsch im Kindergarten
}

\author{
Mathilde Gyger (Liestal)
}

\begin{abstract}
According to the results of the Programme for International Students Assessment (PISA) the linguistic competencies of Swiss students need to be improved. Currently, there is a strong tendency to ban local Swiss-German dialects from Swiss schools, including nursery-schools, and to replace them by Standard High-German more or less completely.

Since parts of the Swiss-German speaking population have strong reservations about HighGerman or at least the use of High-German in nursery-schools, school authorities look for scientific support. Indeed linguistic research has shown favourable results for the pre-school use of High-German: German speaking children are encouraged to use High-German freely and spontaneously. Migrant children benefit regarding fluency and proficiency in German as a second language.
\end{abstract}

The following article comments on some steps taken by school-authorities and summarizes the current discussions on the part of educational and linguistic research in Switzerland.

\section{$1 \quad$ Einleitung}

Hochdeutsch als Unterrichtssprache ist durch den 2003 von der EDK Plenarversammlung verabschiedeten "Aktionsplan PISA 2000" zu einem zentralen Thema der angewandten Linguistik und Sprachdidaktik geworden. Die Schulbehörden bekennen sich zu einer neuen Gewichtung: Aktualisierte Lehrpläne und interkantonale Vereinbarungen postulieren die prioritäre Förderung der Standardsprache "ab Beginn Kindergarten und während der ganzen obligatorischen Schulzeit" (Kanton Solothurn 2006: 7), den weitgehenden Verzicht auf die Mundart (Zürcher Lehrplanüberarbeitung, Bachmann/Peyer 2004: 15) oder formulieren wie der Kanton Nidwalden gezielte Empfehlungen zu Standardsprache im Kindergarten (2004). In zahlreichen Gemeinden werden Pilotversuche auf der Vorschulstufe durchgeführt.

Das Thema lässt auch die Öffentlichkeit nicht unberührt. Im Internet finden sich lesenswerte Blogs ${ }^{1}$ und Leserbriefe zu Zeitungsberichten ${ }^{2}$, die von einer beträchtlichen Skepsis zeugen. Es ist vor allem die Befürchtung des Verlusts mundartlich überlieferten Kulturguts, welche die Gegner auf den Plan ruft. Hochdeutsch im Kindergarten scheint der identitätsstiftenden Rolle einer frühen Begegnung mit schweizerdeutschem Kulturgut - bis anhin ein Kernelement der (vor-)schulischen Sozialisation - zuwider zu laufen.

Der vorliegende Beitrag behandelt aktuelle sprachwissenschaftliche Beiträge zum Thema wie die Dissertation von Landert (2007), die Begleitstudien zu Pilot-Projekten im Kanton BaselStadt (Gyger 2005) und im Kanton Zürich (Bachmann/Sigg 2004) sowie Zwischenergebnisse eines Versuchs im Kanton Basel-Landschaft, der im Jahr 2009 abgeschlossen wird. Im Hinblick auf behördlicherseits eingeleitete Massnahmen gilt der Blick drei Kantonen der Nordwestschweiz - Aargau, Basel-Landschaft und Basel-Stadt -, weil hier dank eines

\footnotetext{
${ }^{1}$ Zum Beispiel http://www.blogwiese.ch/archives/347/.

${ }^{2}$ Zum Beispiel http://classic.facts.ch/dyn/magazin/schweiz/585726.html.
} 
vertieften Einblicks in die laufenden Diskussionen die aktuellen Entwicklungen reflektiert werden können.

Eine Beschränkung auf die Förderung von Deutsch als Zweitsprache ist aufgrund der Sachlage nicht möglich. Die überwiegende Zahl der Studien zum Thema sowie die behördlichen Positionen und Massnahmen sind nicht auschliesslich auf die in sich heterogene Zielgruppe der Kinder mit Migrationshintergrund nicht-deutscher Erstsprache ausgerichtet, denn die Förderung von Hochdeutsch im Kindergarten soll allen Kindern zugute kommen.

\section{Theoretische Positionen und Zielsetzungen}

Aus sprachwissenschaftlicher Sicht ist die Neuorientierung der Schule im Hinblick auf die Standardsprache nachvollziehbar und begründet. Mit Berthele (2004) bzw. Burger/Häcki Buhofer (1998) und Burger/Häcki Buhofer (1994) darf erinnert werden, dass vor Schuleintritt positive oder "zumindest neutrale Einstellungen zur Standardsprache" vorliegen, der Erwerb der gesprochenen Standardsprache mit dem Schuleintritt gehemmt wird und die Abneigung gegenüber dem Hochdeutschsprechen offensichtlich mit der Einstellung der Lehrpersonen zusammenhängt (cf. Berthele 2004: 124). Ziel aktueller Studien ist es festzustellen, ob dies für den Kindergarten gleichermassen gilt.

Arbeiten der Schweizer Linguistik aus der Epoche kurz vor und nach PISA (z. B. Ostermai 2000, Schmidlin 1999, Schneider 1998) weisen auf Bereiche der Sprachkompetenz hin, in denen sich der Effekt des Hochdeutschkindergartens auf Standarderwerb von Kindern mit Deutsch als Erstsprache positiv bemerkbar machen könnte, etwa beim Wortschatz, wo Schneider (1998: 230f.) zeigt, dass es in Ermangelung eines ausgebauten Dialektlexikons und fehlenden Kontrastes zur Standardsprache bis in die zweite Klasse zu einem gemeinsamen Lexikon bzw. zu negativem Transfer aus der Mundart ins Hochdeutsche kommen kann ("gesein"). Sprachdidaktische Schwerpunkte des Kindergartens müssten in diesem Fall bei der Schulung des Sprachbewusstseins und der Verbreiterung des standardsprachlichen Lexikons liegen.

Schmidlin (1999: 276f.) hat beim mündlichen Erzählen die Überlegenheit Deutscher gegenüber Schweizer Kindern in einigen Bereichen bis ins 5. Schuljahr festgestellt, hat aber gleichzeitig nachgewiesen, dass beim Schriftspracherwerb bzw. bei schriftlichen Nacherzählungen keine Standarderwerbsverzögerung vorliegt. Im Gegenteil: Texte von Schweizer Kindern zeigen deutlichere Merkmale der Literalität. Die durch die "Normen der Schriftsprache hervorgerufene grammatikgebundene Übersensibilisierung", die Kinder aus der Deutschschweiz nach Ostermai (2000: 322) im Vergleich zu Kindern aus Südbaden zeigen, ist für die Beeinträchtigung eines "flüssigen und eloquenten" Sprachstils verantwortlich. Hier könnte der Kindergarten durch die bewusste Pflege eines spontanen und informellen Umgangs mit der Standardsprache Positives bewirken, allerdings nur dann, wenn "gewisse Regelzwänge im verbalen Bereich" gelockert werden (ibd.: 323).

Insgesamt ist aufgrund dieser Ergebnisse zu erwarten, dass Deutschschweizer Kinder vor allem im Bereich der Mündlichkeit von einem früh im Kindergarten geförderten vollwertigen, alltagstauglichen und nicht normorientierten Hochdeutsch nachhaltig profitieren könnten. Entsprechend lauten auch einige der Hypothesen von Landert (2007: 21).

Die Zielsetzung von Bachmann/Sigg (2004: 3) ist somit treffend formuliert:

Am Ende der Kindergartenzeit, so die Zielsetzung des Hochdeutsch-Kindergartens, verwenden die Kinder das gesprochene Hochdeutsch ganz selbstverständlich als Umgangs- und Unterrichtssprache und sind, in dieser Hinsicht, gut gerüstet für den Übertritt in die Primarschule. Erwartet wird darüber hinaus, dass der Hochdeutsch-Kindergarten die Kinder in ihrer Freude am Hochdeutschsprechen unterstützt und auch die rezeptiven Fähigkeiten der Kinder fördert. 
Was für Kinder mit mundartlichem Deutsch als Erstsprache gilt, sollte ebenso auf Kinder mit Migrationshintergrund und nicht-deutscher Erstsprache zutreffen. Gleichwohl stehen sich auch hier unterschiedliche Positionen gegenüber, die je nach sprachtheoretischer Ausrichtung dem sprachlichen Input und seiner Qualität (eine oder mehrere Varietäten bzw. Register) unterschiedliches Gewicht beimessen. Namhafte Arbeiten zum Thema der Sprachförderung von Kindern mit Migrationshintergrund stufen die Bedeutung der Varietät Standardsprache für die Sprachförderung als zweitrangig ein (cf. z. B. Penner/Wymann 2003).

Die Forschung zur interkulturellen Pädagogik und Mehrsprachigkeit in der Schule (z. B. Kronig/Haeberlin/Eckhart 2000) beschäftigt sich vor und nach PISA mit geeigneten (Ein-)Schulungsmodellen für Migrantenkinder und mit der Frage des Umfangs von zusätzlichem Stützunterricht in der deutschen Sprache - unbesehen der Varietät, in der er erteilt wird - sowie mit der Frage der Förderung der Erstsprache. Müller (1997: 91) greift die Unterscheidung von Cummins (1979) zwischen kommunikativer Alltagskompetenz in der Lokalsprache (BICS) und kognitiv anspruchsvolleren und komplexeren Sprachfähigkeiten in situationsunabhängiger, formeller Kommunikation (CALP) auf und weist auf dessen These der Bedeutung von CALP-Fähigkeiten in der Herkunftssprache L1 hin, die für den Aufbau in der Zweit- oder Zielsprache L2 entscheidend sind. Er ergänzt diese These durch den Aspekt des schulsprachlichen Selbstvertrauens: "[...] die intellektuell-sprachliche Leistungsfähigkeit und das sichere Wissen der SchülerInnen um diese Leistungsfähigkeit [...]" (ibd.: 283) als mit entscheidendem Erfolgsfaktor. Penner/Wymann (2003: 6) gehen - explizit unter Berufung auf die Ergebnisse der PISA-Studie 2000 - davon aus, dass die sprachliche Förderung in Kindergärten nicht darum für fremdsprachige Kinder unzureichend ist, weil Dialekt gesprochen wird, sondern weil sie sich auf "grundlegende, situationsgebundene Kommunikationsfähigkeiten für den Alltag" konzentriert und die "situationsunabhängige, logische Sprache mit höherer Abstraktion und komplexen Strukturen" vernachlässigt.

Die Anzahl von Zweitspracherwerbsstudien, welche der konkreten Auswirkung des dialektalen Inputs auf den Erwerb einer Zweitsprache gewidmet sind, ist vor allem ausserhalb des deutschen Sprachraums beachtlich (cf. Ellis 2003: 243). Vertreter der Didaktik des Deutschen als Fremdsprache (DaF) weisen darauf hin, dass "durch das Sprechenlernen des Dialekts [...] Ressourcen gebunden [werden], die mit weit mehr Gewinn zum Ausbau produktiver Fertigkeiten in der Standardsprache oder auch für das Erlernen einer weiteren Fremdsprache genutzt werden könnten" (Studer 2002: 125). Dialektkompetenzen gehören nach der Definition des Europäischen Referenzrahmens "zu den höheren und höchsten Niveaus, die LernerInnen in der Fremdsprache erreichen" (ibd.: 129).

Ähnlich lautet das Fazit von Bühlmann (2001): In seiner Studie im Auftrag der Schweizerischen Flüchtlingshilfe stellt Bühlmann 20 ExpertInnen und eine Flüchtlingsgruppe aus Aarau die Frage "Sollen Flüchtlinge Schweizerdeutsch lernen?" und bilanziert: "Hochdeutsch sollte chronologisch an erster Stelle kommen und geniesst gegenüber dem Dialektunterricht ganz klar Priorität. Zuerst sollen die Kenntnisse in der Standardsprache gefestigt werden" (ibd.: 26).

Ziberi-Luginbühl (1999: 15) setzt im Umsetzungsbericht zum NFP 33 hochdeutschdialektales Code-switching im Klassenzimmer und lernersprachlichen Mischcode in ursächliche Verbindung zueinander:

Die gut integrierten mehrsprachigen Kinder haben mit diesem sprachlichen Verhalten [des Code-switchings der Lehrperson] wenig Schwierigkeiten, weil sie sensibel sind für die Regelhaftigkeit von Code-switches. Für die Kinder mit geringer Deutsch-Kompetenz sind die Funktionen der Code-switches der Lehrkraft zwischen Mundart und Hochdeutsch hingegen hochgradig verwirrend, so dass sie auch in der Literalität (beim Vorlesen und Schreiben) die beiden Formen vermischen. 
Aktuelle Forschungsergebnisse bestätigen diesen Befund. Landert (2007: 337) stellt im Zusammenhang mit Selbstkorrekturen ihrer Probandinnen und Probanden beim Hochdeutschsprechen fest:

\begin{abstract}
Während mehrsprachige Kinder oft Mühe mit der Unterscheidung der beiden Varianten hatten, konnten die Kinder mit Erstsprache Schweizerdeutsch besser entscheiden, in welchen Situationen sie welche Varietät sprechen und hören und welche Varietät sie bevorzugen. Die Diglossiesituation in der Deutschschweiz stellt also vor allem mehrsprachige Kinder vor eine grosse Herausforderung: Sie lernen Schweizerdeutsch und Hochdeutsch vielfach nahezu parallel und haben deshalb oftmals Schwierigkeiten mit der Differenzierung der beiden Sprachformen.
\end{abstract}

Aus dieser Perspektive verbinden sich mit dem Gebrauch der Standardsprache im Kindergarten für die Gruppe der Kinder mit Migrationshintergrund nicht-deutscher Erstsprache die Zielsetzungen der Entlastung des Zweitspracherwerbs, die Vermittlung einer positiven Einstellung zur Standardsprache als Affekt-, Spontan- und Beziehungssprache, des flüssigen, eloquenten Sprechens und den Möglichkeiten der rezeptiven und produktiven Unterscheidung zwischen den Varietäten.

\title{
3 Behördliche Massnahmen und Pilotversuche
}

In der Deutschschweiz hat man in den letzten Jahren begonnen, den Unterricht in Versuchsklassen des Kindergartens teilweise oder vollständig auf Hochdeutsch umzustellen. Die Umstellung auf die Standardsprache kann konkrete Massnahmen folgender Art bedeuten:

- Eine vollständige Umstellung des Unterrichts und aller Kommunikation zwischen Lehrpersonen und Kindern auf Hochdeutsch;

die Verwendung der Standardsprache in bestimmten wiederkehrenden Zeitgefässen, z. B. Halbtagen;

- eine (fiktive) Partnerorientierung, d. h. regelmässiger Einsatz einer ausschliesslich Hochdeutsch sprechenden Figur;

- Bindung an bestimmte Unterrichtssituationen, beispielsweise den geführten Ganzklassenunterricht im Kreis;

- Textbezug und -reproduktion: Hochdeutsch erzählte Geschichten, das Einprägen von Versen und Liedern in der Standardsprache.

Weitere Massnahmen betreffen die Weiterbildung und Unterstützung der Lehrpersonen oder die Entwicklung von Handreichungen und Lehrmitteln zum Thema (z.B. Bachmann/Neugebauer 2007).

Drei Kantone der Nordwestschweiz - Basel-Landschaft, Basel-Stadt und Solothurn bekennen sich seit 2006 zu der interkantonalen Vereinbarung der Erziehungsdirektoren und Erziehungsdirektorinnen der Kantone Basel-Landschaft, Basel-Stadt, Bern Freiburg, Solothurn und Wallis (cf. Kanton Solothurn 2006). Neben der Einführung des Französischunterrichts im 3. und des Englischunterrichts im 5. Schuljahr schreibt die Vereinbarung fest (ibd.: 7): "Die lokale Landessprache (Standardsprache) wird ab Beginn Kindergarten und während der gesamten obligatorischen Schulzeit prioritär gefördert." Obwohl der Kanton Aargau dieser interkantonalen Vereinbarung nicht beigetreten ist, positioniert auch er sich im Hinblick auf die Standardsprache klar in diesem Sinn.

Im Kanton Aargau nahmen 2004 bis 200630 Lehrpersonen des Kindergartens an der Versuchsphase "Mundart und/oder Standardsprache im Kindergarten" teil. Jedes Team entwickelte im Laufe des Versuchs eine individuelle Form und wählte das methodischdidaktische Vorgehen selbst. Die Unterrichtsorganisation konnte fixe Phasen, etwa Hochdeutsch-Halbtage, einen situationsbezogenen Gebrauch, etwa beim Basteln, definierte 
Örtlichkeiten, Räume, ausgewählte Spielorte oder eine Bindung an bestimmte Textsorten, z. B. Bilderbücher oder Figuren definieren. Der Versuch galt explizit der Förderung aller Kinder unabhängig von deren Erstsprache. Er beinhaltete eine fachliche Begleitung der Lehrpersonen und führte zu einer Dokumentation, die in eine Handreichung in der Reihe "Schulpraxis konkret" einfliessen wird. Die Dokumentation hält fest, dass Sprachverwirrungen und Mischungen zu vermeiden seien, da sie sogar von den Kindern selbst moniert werden. Ziel von Hochdeutsch im Kindergarten solle sein, dass eine ungezwungene, funktionstüchtige, vielseitig verwendbare und ausbaufähige Umgangssprache gelernt und damit der Grundstein für die Entwicklung komplexerer Sprachfähigkeiten gelegt werde. Eine ganzheitliche pädagogische Beziehungsarbeit in der Standardsprache biete spontanen und affektiven Elementen genügend Raum, da diese für den Spracherwerb wichtig sind. Auf keinen Fall solle die Standardsprache frühzeitig auf einen Lerngegenstand oder eine Schulsprache reduziert werden, in der ausschliesslich unpersönliches Wissen erarbeitet und abgefragt werde. - Der Kanton Aargau wird demnächst eine Revision des Lehrplans verabschieden, die der Standardsprache im Kindergarten mehr Gewicht verleiht.

Im Kanton Basel-Landschaft beschloss der Bildungsrat im Jahr 2003 eine StufenlehrplanErweiterung im Sinne einer Weisung zum Gebrauch der deutschen Standardsprache im Kindergarten. Die zugrunde liegende Weisung des Erziehungsrates vom 20.11.2002 hält fest: "Im Kindergarten werden sowohl die mundartliche Sprachkompetenz wie auch die Bereitschaft, die deutsche Standardsprache zu erlernen, gefördert." Die Weisung schlägt vor, dass beispielsweise Vorlesesequenzen zu Hör- und Verstehenserfahrungen führen sollen, während in Erzähl- und Spielsequenzen der aktive Gebrauch der Standardsprache geübt werden soll.

Um zu prüfen, in welcher Art und Weise die Vorgaben des erweiterten Stufenlehrplans im Kindergarten am gewinnbringendsten umzusetzen sind, führt die Stadt Liestal das wissenschaftlich begleitete Pilotprojekt "Deutsch-Standard im Kindergarten" durch. Seit Beginn des Schuljahres 2005/06 wird die Verwendung der Standardsprache in drei unterschiedlichen Modellen erprobt: In einem der drei Projektkindergärten wird in denjenigen Unterrichtssequenzen, in denen alle Kinder gemeinsam gefördert werden, im so genannten geführten Teil, Standardsprache und in anderen Teilen, etwa im Freispiel, Mundart gesprochen. Instruktionen erfolgen in der Standardsprache, Geschichten und ausgewählte Verse und Lieder werden in der Standardsprache vermittelt. Da sich geführte und nicht geführte Teile an einem Kindergartenvormittag abwechseln, kann es mehrmals zu einem Wechsel zwischen Mundart und Standardsprache kommen. In einem weiteren Kindergarten wird die Standardsprache in regelmässigen Sequenzen mithilfe einer Leitfigur vermittelt, einer Hasen-Handpuppe, die ausschliesslich Standardsprache spricht. Die Leitfigur wird häufig dann eingesetzt, wenn die Lehrperson etwas Neues einführt oder eine Geschichte erzählt. Die Handpuppe ist täglich ungefähr 30 Minuten im Einsatz, wobei die Sequenz nicht zwingend am Stück stattfinden muss. Im dritten Projektkindergarten wird ausschliesslich in der Standardsprache unterrichtet, sowohl in der formellen Unterrichtskonstellation als auch in Einzelgesprächen und in spontanen Kommunikationssituationen. Auch auf Ausflügen oder dem Weg ins Turnen sprechen die Lehrpersonen mit den Kindern Standardsprache. Ausnahmen bilden einzelne Verse, Lieder oder Spiele, die ihren starken Authentizitätscharakter einbüssten, würden sie in die Standardsprache "übersetzt" (Fasnacht: Larve, Herbstmesse: Mässmogge, Rösslirytti usw.). Der Förderunterricht Deutsch als Zweitsprache (DaZ), der fremdsprachigen Kindern zweimal wöchentlich während 50 Minuten erteilt wird, findet wie in allen Kindergärten der Stadt Liestal auf Hochdeutsch statt.

Eine Empfehlung für ein bestimmtes der erprobten Modelle wird der Schulrat nach Abschluss des 4-jährigen Projekts und der Begleitstudie dann aussprechen, wenn es sich 
- bereits während der Kindergartenzeit eindeutig vorteilhaft auf die Hochdeutschkenntnisse der Kinder auswirkt und

- auf den Schulerfolg der Kinder bis zum Ende des zweiten Schuljahres einen nachhaltig positiven Einfluss hat.

Da der Zwischenbericht dem Versuch ermutigende Ergebnisse bescheinigt, hat der Schulrat bereits entschieden, dass künftig nachmittags in allen Gruppensituationen Hochdeutsch unterrichtet werden soll (Brian Karaman 2007: 9).

Die Sprachenpolitik des Erziehungsdepartementes des Kantons Basel-Stadt orientiert sich in erster Linie am Gesamtsprachenkonzept des Kantons (2003). Nach Abschluss des wissenschaftlich begleiteten Pilotversuchs "Standardsprache im Kindergarten" mit zwei vollständig in der Standardsprache geführten Kindergärten entschied sich der Kanton BaselStadt für eine weiterführende, zweijährige Erprobungsphase. Im Basler Versuch hatte man anfänglich vor allem Kinder mit Migrationshintergrund im Auge und argumentierte mit der Entlastung des Zweitspracherwerbs und die verstärkte Vorbereitung auf die Schulsprache Hochdeutsch. Aufgrund der Erfolge gelangte man zur Überzeugung, dass die Standardsprache allen Kindern zugutekommen sollte. Auch in Basel ist die sprachliche Heterogenität der Kindergärten unterschiedlich gross. Die weiterführende Erprobungsphase soll der Standardsprache im Kindergarten zu einer breiten Akzeptanz in der Bevölkerung und bei den Lehrpersonen verhelfen. Für das Schuljahr 2006/07 konnten 16 und für das folgende Schuljahr weitere 15 Kindergartenteams für die Erprobungsphase gewonnen werden. In diesen Kindergärten wird der Unterricht vollständig auf Hochdeutsch umgestellt. Die Lehrpersonen erhalten die Möglichkeit, Erfahrungen zu sammeln und von einem auf ihre Bedürfnisse zugeschnittenen Weiterbildungsprogramm zu profitieren. In der Erprobungsphase sollen ausserdem die Aussagen und Empfehlungen der Experten überprüft werden.

\section{$4 \quad$ Wissenschaftliche Studien}

Bereits 2004 haben Bachmann/Sigg die Studie "Hochdeutsch-Kindergarten: Die Chancen des frühen Beginns" vorgelegt, die im Rahmen des Projektes "Förderung der deutschen Standardsprache" der Bildungsdirektion und der Pädagogischen Hochschule Zürich entstand. Die Zürcher Studie befasst sich mit der sprachlichen Förderung von Kindern sowohl deutscher als auch anderer Herkunftssprache. Die Studie dokumentiert die Beobachtungen und Einschätzungen der Lehrpersonen und formuliert Empfehlungen u. a. zum Ausbau des Angebots an Hochdeutschkindergärten.

Grundlage der Studie ist ein Schulversuch mit einem Kindergarten, der in Hochdeutsch geführt wurde. Zwei weiterhin in der Mundart geführte Kindergärten am selben Schulort erlaubten einen Vergleich der Wirkung der Standardsprache, und zwar sowohl in den Kindergärten selbst als auch in gemischten ersten Klassen der Primarschule. Die beteiligten Lehrpersonen wurden mittels eines Fragebogens und eines anschliessenden Interviews zweimal, zum Ende des zweiten Kindergartenjahres und zum Ende der ersten Primarklasse, zur sprachfördernden Wirkung der Standardsprache im Kindergarten auf die für die Stichprobe ausgewählten acht Kinder befragt.

Die Studie interpretiert die Beobachtungen und Einschätzungen der Lehrpersonen und formuliert Empfehlungen: Die Kinder aus dem Hochdeutschkindergarten entwickeln unabhängig von ihrer Ausgangssprache Freude am Hochdeutschsprechen, jedoch nicht auf Kosten der Mundart. Anfangs bevorzugen Kinder mit Schweizerdeutsch sprechenden Eltern die Mundart als Affekt- und Spontansprache. Aber auch diese Kinder verwenden die Standardsprache bei längerer Verweildauer im Kindergarten zunehmend und konsequent. Dieser Effekt wirkt nachhaltig in die erste Klasse der Primarschule hinein (Bachmann/Sigg 
2004: 32). Kinder aus dem Hochdeutschkindergarten legen ausserdem eine unbefangenere Probierhaltung an den Tag und entwickelten ein grösseres Sprachbewusstsein. Allerdings profitieren Kinder aus einem fremd- oder mehrsprachigen Elternhaus in dieser Hinsicht stärker als solche mit Schweizerdeutsch sprechenden Eltern.

Die Empfehlungen auf diesem Hintergrund fordern unter anderem,

- dass das Angebot an Hochdeutschkindergärten ausgebaut und Hochdeutsch auch im Rahmen anderer Projekte als selbstverständliche Unterrichtssprache etabliert werden solle.

- Parallel dazu sei das Weiterbildungsangebot für Lehrpersonen und Schulbehörden Sprachförderung in Richtung funktionaler Mehrsprachigkeit - zu stärken.

- In der Ausbildung der Lehrpersonen seien Fragen der Einstellung gegenüber der Standardsprache zu thematisieren.

- Die situationsspezifische Verwendung von Hochdeutsch und Standardsprache sei zu vermeiden und auf die Korrekturpraxis der Lehrpersonen zu achten.

- In behördlichen Weisungen solle man auf offene Formulierungen wie "möglichst viel Hochdeutsch" verzichten, damit die bisherige Praxis nicht tradiert werde.

Die Basler Studie "Standardsprache im Kindergarten" (Gyger 2005) widmet sich ausschliesslich fremd- und mehrsprachigen Kindern mit Migrationshintergrund und rät vor allem zum Gebrauch der Standardsprache in Klassen für Kinder mit geringen Deutschkenntnissen. Die Begleitstudie zu dem vierjährigen Schulversuch wurde vom Erziehungsdepartement des Kantons Basel-Stadt getragen. Sie behandelt einerseits die Erfahrungen der Lehrpersonen und andererseits die Bedeutung der Standardsprache für den Spracherwerb der Kinder.

Der Versuch war als quasi-experimentelle Langzeitstudie mit einer Vergleichs- und einer Versuchsgruppe angelegt. In den Jahren 2001 bis 2003 wurden als Versuchsgruppe zwei Kindergartenklassen in der Standardsprache und als Vergleichsgruppen zwei weitere Kindergärten in der Mundart geführt. In beiden Gruppen lag der Anteil fremd- und mehrsprachiger Kinder mit Migrationshintergrund je nach Erhebungszeitpunkt zwischen 90 und $100 \%$. Im Projekt wurde nur der im Jahr in 2001 eintretende Jahrgang berücksichtigt, weil nur bei ihm der Stand der Zweitsprache Deutsch bei Eintritt erfasst werden konnte. Nach dem Schuleintritt der Kinder im August 2003 wurde die Nachhaltigkeit der Standardsprache für die Weiterentwicklung des Zweitspracherwerbs und den Aufbau schriftsprachlicher Kompetenzen in den ersten zwei Schuljahren dokumentiert.

Die gesamte Stichprobe umfasste bei Abschluss des Projekts 16 Kinder: 8 Kinder der Versuchsgruppe und 8 der Vergleichsgruppe. Die Erhebungen erfolgten in mindestens halbjährlichem Abstand: Die Erstsprachkompetenz der Projektkinder wurde einmalig durch Expertinnen und Experten bzw. Lehrkräfte für Heimatliche Sprache und Kultur (HSK) erhoben. Das familiäre Sprachverhalten und seine Hintergründe, Familiengeschichte und Migration, Mediengewohnheiten, Sozialkontakte, Bildungserfahrungen, wurden anhand eines Fragebogens ebenfalls in der Herkunftssprache in Erfahrung gebracht. Die Quantität und Qualität des Inputs in der Zielsprache Deutsch findet sich in mehrstündigen exemplarischen Tonaufnahmen der Lehrpersonen im Kindergarten "in vivo" dokumentiert. Daten zur Sprachentwicklung der Kinder wurden einerseits in Wortschatz- und Grammatiktests erhoben und ferner im Jahresabstand anhand von Spontansprachproben in Form von Nacherzählung einer Trickfilmgeschichte ("Kleiner Eisbär"). Die Erhebungen fanden in den ersten zwei Projektjahren in der Kommunikationssprache des jeweiligen Kindergartens statt. In den darauf folgenden beiden Schuljahren wurden alle Daten auf Hochdeutsch erhoben. 
Die Sprachproben ergaben bei der Versuchsgruppe mit Standardsprache im Laufe der Kindergartenzeit eine stärkere Zunahme von Sprechaktivität und Wortschatz als bei der Vergleichsgruppe mit Mundart im Kindergarten.

Der Schuleintritt zog in der Versuchsgruppe beträchtliche Umwälzungen nach sich. Bei den Kindern aus einem der beiden Hochdeutschkindergärten nahmen - den sprachlichen Gepflogenheiten in der Schulklasse entsprechend - vorübergehend mundartliche Elemente in der gesprochenen Standardsprache stark zu. Der Schulerfolg fiel hingegen laut Statistik bei beiden Projektgruppen nahezu gleich aus.

Der Schlussberichts zum Basler Projekt zeigt, dass die Standardsprache gegenüber der Mundart im Kindergarten für den Zweitspracherwerb Vorteile bringt, zwar nicht als alleiniger Erfolgsfaktor, aber in Verbindung mit anderen günstigen Gegebenheiten: einer hohen Lernmotivation, z. B. nach kürzlicher Einwanderung aus dem Ausland, einem soliden Fundament in der Erstsprache, intensiven angeleiteten Kontakten mit Deutschsprachigen in der Freizeit oder Unterstützung durch ein bildungsbewusstes Elternhaus. Dies gilt insbesondere für Kinder mit geringen Deutschkenntnissen. Mit konsequentem Hochdeutsch im Kindergarten nehmen Sprechaktivität und Wortschatz stärker $\mathrm{zu}$ und sind der Standardsprache näher. Deutliche Vorteile zeigen sich überraschender Weise auch beim Schriftspracherwerb: in den Bereichen von Leseverstehen, Schreibaktivität und Orthographie.

Die Studie empfiehlt folglich unter anderem

- in Kindergärten, die von Kindern mit geringen Deutschkenntnissen besucht werden, auf die Standardsprache umzustellen und

- eine Begleitung und Weiterbildung der Lehrpersonen vorzusehen.

Landerts Dissertation (2007) Hochdeutsch im Kindergarten? ist als empirische Studie ebenfalls einem hochdeutsch geführten Projektkindergarten in einer Agglomerationsgemeinde nahe der Stadt Zürich gewidmet, der mit einem schweizerdeutsch geführten Kindergarten in der gleichen Region verglichen wird. Landert beobachtete in zwei Erhebungsphasen Schweizerdeutsch-(Sd.-)Kindergarten und 9 Kinder, die den Hochdeutsch-(Hd.-)Kindergarten besuchten. Die Erhebung bestand aus einem Gespräch mit den Kindern und der Nacherzählung einer Bildergeschichte (Landert 2007: 72). Landerts Grundfrage lautet (ibd.: 16): "Erbringen die Kinder des Hd.-Kindergartens bessere Leistungen in ihrer Hochdeutschproduktion als die Kinder des Sd.-Kindergartens?"

Im Folgenden sollen auf eine Auswahl von Ergebnissen im Hinblick auf die sprachliche Leistung eingegangen werden: Textstruktur, Reichhaltigkeit und Flüssigkeit des Sprechens (von Landert beobachtet anhand von Textlänge und Länge der Gesprächsbeiträge bzw. Anzahl gesprochener Wörter, syntaktischer Einheiten und der Breite des Wortschatzes (2007: 86 und 107), die formale Korrektheit anhand ausgewählter phonetischer, morphologischer und lexikalischer Aspekte (2007: 159), und die Einstellung der Lehrpersonen, erfragt in Interviews (2007: 289).

Landert (2007: 338f.) verwirft aufgrund ihrer Ergebnisse die Hypothese, dass die formale Korrektheit durch den Gebrauch von Hochdeutsch im Kindergarten generell zunimmt. Sie stellt jedoch fest, dass die mehrsprachigen Kinder mit geringen Mundartkompetenzen in dieser Hinsicht stärker profitieren als Kinder mit Erstsprache Schweizerdeutsch. Die Begründung lautet: Kinder mit Migrationshintergrund haben weniger Möglichkeiten zum Transfer aus dem Schweizerdeutschen und deren Spracherwerb hängt stärker von standardsprachlichem Input ab. Eine Wechselwirkung zwischen den Input-Qualitäten der Lehrpersonen und den Sprachleistungen der Kinder ist nicht festzustellen, wohl aber bestätigt sich die Annahme, dass sich die positive Einstellung der Lehrpersonen und die "konsequente 
Verwendung" der Standardsprache bei den Kindern deutliche Spuren hinterlässt. Im Hinblick auf die verschiedenen Modelle des Hochdeutschgebrauchs, die aktuell erprobt werden, ist die Bemerkung interessant (ibd.: 343), dass "die Standardsprache in allen Situationen gesprochen werden sollte, weil die Kinder ansonsten Mühe mit der Unterscheidung von Schweizerdeutsch und Hochdeutsch haben und nicht wissen, welche Sprachform aus welchen Gründen gesprochen wird." Landert (ibd.: 344) fasst zusammen:

Die Unterrichtssprache Hochdeutsch im Kindergarten trägt gemäss den Resultaten der vorliegenden Untersuchung nicht wesentlich zur Steigerung der sprachformalen Leistungen in der Standardsprache bei. Es lässt sich aber ein wesentlicher Einfluss auf die Struktur und Reichhaltigkeit des Hochdeutschen feststellen. Ausserdem bringen die Kinder, die einen Hochdeutschkindergarten besuchen, eine hohe Lernmotivation und einen unbefangene(re)n Umgang mit dieser Sprachform und eine sehr positive Einstellung gegenüber der Standardsprache mit. [...] Davon profitieren [...] die mehrsprachigen Kinder als auch die Kinder mit Erstsprache Schweizerdeutsch.

Landert bestätigt im Übrigen den Befund von Gyger (2005): "Genauso wichtig wie die konsequente Verwendung der Standardsprache im Kindergarten ist deren konsequente Weiterverwendung in der Primarschule." (Landert 2007: 344)

Im Jahr 2005 beauftragte die Schule Liestal die heutige Fachhochschule Nordwestschweiz FHNW mit der Begleitung des Projekts "Deutsch-Standard im Kindergarten". Das Projekt hat eine Laufzeit von vier Jahren. Es beinhaltet die Unterstützung und Beratung der am Projekt beteiligten Lehrpersonen und die Ausarbeitung einer empirisch angelegten Begleitstudie, welche die Kinder während des Kindergartens und der anschliessenden zwei Primarschuljahre erfasst. Offizieller Projektbeginn war nach einer einjährigen Vorlaufphase der 1. August 2005. Der Schlussbericht wird im Herbst 2009 erwartet.

Die Begleitstudie dokumentiert die drei Modelle und die Sprachentwicklung aller im August 2005 in die drei Projektkindergärten eingetretenen Kinder über insgesamt vier Jahre. Erfasst wurden 19 Kinder, die im Schuljahr 2005/06 neu eintraten. Die Kinder sind mehrheitlich nicht-deutscher Herkunftssprache. Im September 2005 fanden die ersten Datenerhebungen in Form standardisierter Sprachtests sowie des Erzählen eine Bildergeschichte statt. Bei 17 der 19 an der Untersuchung beteiligten Kinder konnten ausserdem Abklärungen zur Kompetenz in der Herkunftssprache durchgeführt werden. - Nach Schuleintritt der Kinder folgen ab 2007 in den ersten beiden Schuljahren weitere Sprachstandserhebungen zur mündlichen und schriftlichen Kompetenz in der Standardsprache. Zudem wird der Schulerfolg der Kinder in Zusammenarbeit mit den Lehrpersonen der Primarschule ermittelt. Daneben werden auch die Lehrpersonen über ihre Spracheinstellungen und ihre Unterrichtserfahrungen befragt und in ihrem Sprachverhalten beobachtet.

Eine Kurzfassung der wichtigsten Zwischenergebnisse stellt Leuenberger (2007) in den Baselandschaftlichen Schulnachrichten vor: Betrachtet man die Entwicklung in den einzelnen Kindergärten, zeigt sich, dass die Kinder, die am meisten standardsprachlichen Input erhalten, beim aktiven wie passiven Wortschatz die grössten Fortschritte erzielen. Jene Kinder, die "nur" im geführten Unterricht oder beim Auftreten der Leitfigur (Handpuppe) mit der Standardsprache konfrontiert werden, erzielen geringere Fortschritte. Um Sprechaktivität und Erzählkompetenzen beobachten zu können, wurden jedem Kind zum Schluss der Befragung sechs Karten in Form einer Bildergeschichte gezeigt. Im Zeitvergleich zeigt sich, dass wiederum diejenigen Kinder, die ausschliesslich in der Standardsprache unterrichtet werden, die grössten Fortschritte erzielen. Leuenberger (ibd.: 14) schlussfolgert:

Im Wissen darum, dass Sprachenlernen nicht monokausal und unabhängig von sozioökonomischen und zahlreichen anderen Faktoren zu erklären ist, lassen die vorliegenden Ergebnisse den Schluss zu, dass der konsequente Gebrauch der Standardsprache bei Kindern 
mit geringen Deutschkompetenzen - diese Kinder 'starten' immer auf niedrigstem Niveau und erreichen nie ein höchstes - die grössten Fortschritte bewirkt. Sowohl beim passiven und aktiven Wortschatz als auch in Bezug auf die Sprechaktivität kann dieser Befund für jene Kinder, die den grösstmöglichen standardsprachlichen Input erhalten, nachgewiesen werden. Ist ein gewisser Sprachstand erreicht, scheint sich die günstige Wirkung abzuschwächen. $\mathrm{Ob}$ und welche Effekte dies auf Orthographie, Leseverstehen, Schreibfreude, Wortschatzerweiterung, Ausbau der Lernergrammatik und damit auf den Schulerfolg haben wird, muss abgewartet werden.

\section{$5 \quad$ Fazit und offene Fragen}

Nur ungern und vielfach ideologisierend hat sich das öffentliche Bewusstsein bisher mit den Vorteilen von Hochdeutsch im Kindergarten auseinandergesetzt. Sprachwissenschaft und Sprachdidaktik sehen inzwischen klarer: Hochdeutsch im Kindergarten ist im Hinblick auf Aspekte der sprachformalen Sicherheit beim Erwerb von Deutsch als Zweitsprache vor allem für Kinder mit geringen Deutschkenntnissen vorteilhaft (cf. Gyger 2005; Landert 2007).

Überraschenderweise zeigen sich positive Effekte auch beim Schriftspracherwerb - und zwar bei der Orthographie, in einem reichhaltigeren Wortschatz und grösserer Schreibaktivität. Unbefangenheit, erhöhte Sprechaktivität und positivere Einstellungen zur Standardsprache sind bei allen Kindern, einschliesslich derer mit Schweizerdeutsch als Erstsprache, festzustellen.

Wie konsequent und unbefangen Hochdeutsch in der Primarschule anschliessend an den Hochdeutschkindergarten gesprochen wird, ist für den Erhalt und Ausbau der Sprachkompetenz entscheidend. Erste Ergebnisse weisen darauf hin, dass eine vollständige einer teilweisen Umstellung auf die Standardsprache vorzuziehen ist. In dieser Hinsicht soll die Begleitstudie zum Liestaler Versuch mehr Klarheit schaffen.

Die folgenden beiden Fragestellungen konnten in keiner der bisherigen Studien befriedigend aufgearbeitet werden: Vertreterinnen und Vertreter derjenigen Instanzen, welche für die logopädische und heilpädagogische Förderung zuständig sind, betonen, dass die Bedeutung der Standardsprache im Kindergarten für Kinder mit besonderen Lernbedürfnissen dringend mehr Beachtung finden sollte. An dieser Stelle wäre eine interdisziplinäre Diskussion sehr zu begrüssen. Die Deutschdidaktik fordert in jüngerer Zeit den Paradigmenwechsel vom schriftlichkeitsnahen zum mündlichkeitsnahen Hochdeutschsprechen in der Schule. Während diese Abkehr von unangemessenen Regelzwängen im Unterricht zur Entspannung der Sprachsituation in Schule und Kindergarten und zur Verbesserung der Spracheinstellung beiträgt, fragt es sich, ob sie für den Erwerb von Deutsch als Zweitsprache in Hinblick auf die Förderung sprachformaler Kompetenzen - vor allem in den Bereichen von Morphologie und Syntax - ebenso vorteilhaft ist. Möglicherweise bedarf es hier - zumindest in den Anfangsstadien des Erwerbs von Deutsch als Zweitsprache - einer Verbindung der Ansprüche: einer gelockerten Haltung im Einklang mit einer erhöhten sprachformalen Korrektheit.

\section{Literaturangaben}

Bachmann, Thomas/Neugebauer, Claudia (eds.) (2007): Handbuch Hochdeutsch. Grundlagen, Praxisberichte und Materialien zum Thema Hochdeutschsprechen in der Schule. Zürich.

Bachmann, Thomas/Peyer, Anne (2004): Lehrplanüberarbeitung im Bereich deutsche Standardsprache. Schlussbericht zuhanden der Bildungsdirektion des Kantons Zürich. http://www.bildungsdirektion.zh.ch/internet/bi/de/BR/brb_02_05.SubContainerList.SubCo ntainer3.ContentContainerList.0039.DownloadFile.pdf, Stand 17.12.2007. 
Bachmann, Thomas/Sigg, Marianne (2004): Hochdeutsch-Kindergarten: Die Chancen des frühen Beginns. Bericht zur explorativen Studie "Hochdeutsch im Übergang zwischen Kindergarten und Primarschule". Zürich.

Berthele, Raphael (2004): "Vor lauter Linguisten die Sprache nicht mehr sehen - Diglossie und Ideologie in der deutschsprachigen Schweiz". In: Christen, Helen (ed.): Dialekt, Regiolekt und Standardsprache im sozialen und zeitlichen Raum. Beiträge zum 1. Kongress der Internationalen Gesellschaft für Dialektologie des Deutschen, Marburg/Lahn, 5.-8. März 2003. Wien: 111-136.

Brian Karaman, Dorothée (2007): "Vorsprung durch Bildung". Basellandschaftliche Schulnachrichten 3/2007: 9.

Bühlmann, Felix (2001): Sollen Flüchtlinge Schweizerdeutsch lernen? Eine explorative Studie $z u$ den Vorteilen von Schweizerdeutschunterricht und Hochdeutschunterricht für Flüchtlinge mit Status B. Studie erstellt im Auftrag der Schweizerischen Flüchtlingshilfe. unveröff. Ms.

Burger, Harald/Häcki Buhofer, Annelies (1998): Wie Deutschschweizer Kinder Hochdeutsch lernen: der ungesteuerte Erwerb des gesprochenen Hochdeutschen durch Deutschschweizer Kinder zwischen 6 und 8 Jahren. Stuttgart. (= ZDL Beihefte 98).

Burger, Harald/Häcki Buhofer, Annelies (eds.) (1994): Spracherwerb im Spannungsfeld von Dialekt und Hochsprache. Bern etc.

Cummins, John (1979): "Linguistic Interdependence and the Educational Development of Bilingual Children". Review of Educational Research 2/49: 222-251.

EDK - Schweizerische Konferenz der kantonalen Erziehungsdirektoren (2003): Aktionsplan "PISA 2000"-Folgemassnahmen. Bern. http://www.edk.ch/pdf_downloads/monitoring/ aktplanpisa2000_d.pdf, Stand 17.12.2007.

Ellis, Rod (2003): The Study of Second Language Acquisition. $10^{\text {th }}$ ed. Oxford.

Gyger, Mathilde (2004): "Standardsprache im Kindergarten - eine neue sprachdidaktische Herausforderung". Bulletin suisse de linguistique appliquée 79: 123-147.

Gyger, Mathilde (2005): Projekt Standardsprache im Kindergarten. Schlussbericht. Basel. http://kg.edubs.ch/aktuell/pss_gesamtbericht.pdf, Stand 17.12.2077.

Gyger, Mathilde/Heckendorn-Heinimann, Brigitte (2007): "Schulentwicklungsprojekt der Schule Liestal: Deutsch-Standard im Kindergarten". Basellandschaftliche Schulnachrichten 3/2007: 6-9.

Kanton Basel-Stadt (2003): Ein Gesamtsprachenkonzept für die Schulen Basel-Stadt. http://www.edubs.ch/die_schulen/projekte/archiv/gesamtsprachenkonzept/index.pt.

Kanton Nidwalden (2004): Kindergarten - Hinweise zur Verwendung des Hochdeutsch. Stans. http://www.nw.ch/dl.php/de/20051221150514/kg_hochdeutsch.pdf, Stand 17.12. 2007.

Kanton Solothurn (2006): Beitritt des Kantons Solothurn zur interkantonalen Vereinbarung der Erziehungsdirektoren und Erziehungsdirektorinnen der Kantone Basel-Landschaft, Basel-Stadt, Bern, Freiburg, Solothurn und Wallis zur Einführung des Französischunterrichts ab dem 3. Schuljahr und des Englischunterrichts ab dem 5. Schuljahr sowie zur gemeinsamen Entwicklung des Fremdsprachenunterrichts (FEUV). Botschaft und Entwurf des Regierungsrates an den Kantonsrat von Solothurn vom 14. August 2006. (= RRB Nr. 2006/1511).

http://www.old.so.ch/extappl/rrb/daten/rrb2006/0814/000000565506_2006_1511.pdf, Stand 17.12.2007.

Kronig, Wilfried/Haeberlin, Urs Eckhart (2000): Immigrantenkinder und schulische Selektion. Pädagogische Visionen, theoretische Erklärungen und empirische Untersuchungen zur Wirkung integrierender und separierender Schulformen in den Grundschuljahren. Bern.

Landert, Karin (2007): Hochdeutsch im Kindergarten? Eine empirische Studie zum frühen Hochdeutscherwerb in der Deutschschweiz. Bern etc. 
Leuenberger, Petra (2007): "Begleitstudie zum Liestaler Projekt 'Deutsch-Standard im Kindergarten' - Zwischenergebnisse aus zwei Kindergartenjahren". Basellandschaftliche Schulnachrichten 3/2007: 10-14.

Müller, Romano (1997): Sozialpsychologische Grundlagen des schulischen Zweitspracherwerbs bei MigrantenschülerInnen. Theoretische Grundlagen und empirische Studien bei zweisprachigen und einsprachigen SchülerInnen aus der 6.-10. Klasse in der Schweiz. Aarau.

Neugebauer, Claudia/Nodari, Claudio (1999): "Aspekte der Sprachförderung". In: Gyger, Mathilde/Heckendorn-Heinimann, Brigitte (eds.): Erfolgreich integriert? Fremd- und mehrsprachige Kinder und Jugendliche in der Schweiz. Bern: 160-175.

Ostermai, Guido (2000): Sprachvariation im Grenzbereich. Eine Untersuchung zu Standardsprache nordwestschweizerischer und südbadischer PrimarschülerInnen. Aarau. (= Sprachlandschaften 24).

Penner, Zvi (2003): Neue Wege der frühen Sprachförderung von Migrantenkindern. Forschung für die Praxis. Bern.

Penner, Zvi/Wymann, Karin (2003): "Deutsch sprechen lernen genügt noch nicht". 4 bis 8 Fachzeitschrift für Kindergarten und Unterstufe 1: 5-7.

Riemer, Claudia (1997): Individuelle Unterschiede im Fremdsprachenerwerb. Die Wechselwirksamkeit ausgewählter Einflussfaktoren. Hohengehren.

Schmidlin, Regula (1999): Wie Deutschschweizer Kinder schreiben und erzählen lernen. Textstruktur und Lexik von Kindertexten aus der Deutschschweiz und aus Deutschland. Tübingen/Basel. (= Basler Studien zur deutschen Sprache und Literatur 79).

Schneider, Hansjakob (1998): "Hochdeutsch - das kann ich auch". Der Erwerb des Hochdeutschen in der deutschen Schweiz: Eine Einzelfallstudie zur frühen mündlichen Sprachproduktion. Bern.

Studer, Thomas (2002): "Dialekte im DaF-Unterricht? Ja, aber... Konturen und Konzepte für den Aufbau einer rezeptiven Varietätenkompetenz". Linguistik online 10, 1/02: 113-131.

Werlen, Erika/Ernst, Karl (1994): "Dialektale und hochsprachliche Kommunikationskultur von Schulkindern. Hypothesen und Zugänge." In: Burger, Harald/Häcki Buhofer, Annelies (eds.): Spracherwerb im Spannungsfeld von Dialekt und Hochsprache. Bern: 215-241.

Ziberi-Luginbühl, Johanna (1999): Zweitsprachunterricht im obligatorischen Schulsystem. Umsetzungsbericht. Bern/Aarau. 